\title{
MiR-19b-3p Regulates MAPK1 Expression in Embryonic Fibroblasts from the Great Tit (Parus Major) Under Hypoxic Conditions
}

\author{
Xiumin Chen ${ }^{a, b, c}$ Yanhua Qu ${ }^{a} \quad$ Yalin Cheng ${ }^{a} \quad$ Jing Wang $^{\mathrm{a}} \quad$ Xiaohua Lei $^{\mathrm{a}}$ \\ Gang Song $^{a}$ Huishan Zhang ${ }^{\mathrm{a}}$ Haitao Wange Fumin Lei ${ }^{\mathrm{a}, \mathrm{c}, \mathrm{d}}$
}

aKey Laboratory of Zoological Systematics and Evolution, Institute of Zoology, Chinese Academy of Sciences, 1 Beichen West Road, Chaoyang District, Beijing, 'Institute of Special Animals and Plants Sciences, Chinese Academy of Agricultural Sciences, Changchun, University of the Chinese Academy of Sciences, Beijing, ${ }^{d}$ Center for Excellence in Animal Evolution and Genetics, Chinese Academy of Sciences, Kunming, eNortheast Normal University, Changchun, China

\section{Key Words}

miR-19b-3p • MAPK1 • Embryonic Fibroblasts • Hypoxic Adaptation

\begin{abstract}
Background/Aims: Genomic adaptations to high altitudes have been well studied in the last several years; however, the roles of microRNAs (miRNAs), which are essential modulators of a variety of genes and key cellular processes, have rarely been explored. Here, we explored the interactions between miRNAs and their target genes as an adaptation to high altitude in an avian species, the great tit (Parus major), which is widely distributed across the Eurasian continent at altitudes between $4500 \mathrm{~m}$ and sea level. Because the MAPK signaling pathway plays a crucial role in the hypoxia response in the great tit, we chose MAPK1 as a target candidate gene. Methods: We established a great tit embryonic fibroblast line and subsequently studied the relationship between miRNA-19b-3p and MAPK1 in normoxia and hypoxia groups. Meanwhile, the great tit embryonic fibroblasts (GEFs) were treated or transfected with miR-19b-3p mimics, inhibitors, or si-MAPK1, and their proliferation was subsequently assessed using the MTT assay. The expression of the miRNAs and MAPK1 was measured by real-time PCR and Western blotting. Results: We identified 14 miRNAs in the cardiac tissues of great tits that are related to hypoxia adaptation. MAPK1 binds only to miR$19 \mathrm{~b}-3 \mathrm{p}$ of the 14 miRNAs predicted by both TargetScan and miRanda software. Specifically, we validated the computational prediction of miR-19b-3p binding to the 3'UTR of MAPK1 using a luciferase reporter assay. Our results show that miR-19b-3p promotes GEFs proliferation and up-regulates MAPK1 expression. Moreover, miR-19b-3p mimics and MAPK1 knockdown induce GEFs apoptosis and regulate the cell cycle under hypoxic conditions. Conclusions: Our study is the first to describe an important miRNA-mediated regulatory mechanism of high altitude adaptation in a non-model wild songbird and highlights the importance of studies on miRNA-mediated mechanisms of hypoxic adaptations in other animals

(C) 2018 The Author(s)

Published by S. Karger AG, Basel




\section{Cellular Physiology Cell Physiol Biochem 2018;46:546-560 \\ \begin{tabular}{ll|l} 
and Biochemistry & $\begin{array}{l}\text { DOI: 10.1159/000488621 } \\
\text { Published onlIne: April 04, } 2018\end{array}$ & $\begin{array}{l}\text { C } 2018 \text { The Author(s). Published by S. Karger AG, Basel } \\
\text { www.karger.com/cpb }\end{array}$ \\
\hline
\end{tabular}}

\section{Introduction}

MicroRNAs (miRNAs) are a group of non-coding RNAs ( $\sim 22 \mathrm{nt})$ that silence gene expression by binding to the coding region of target mRNAs or to the 3' UTR to decrease mRNA stability or prevent protein translation. miRNAs are essential modulators of a variety of genes and key cellular development processes $[1,2]$. miRNAs also have an important relationship with hypoxia. As shown in a previous study, miR-190 appears to be a positive regulator of $\mathrm{Ca}^{2+}$ influx in arterial smooth muscle cells and plays an important role in hypoxic pulmonary vascular constriction [3]. Hypoxia significantly modifies the miRNA profile in the heart and liver, which regulate diverse cellular and molecular functions. Therefore, it is possible to alter hypoxia/ischemic pathophysiology in the heart [4] or liver [5]. miR126 [6], miR-139 [7] and miR-208 [8] have been shown to be especially protective from the effect on hypoxia/ischemia- or hypoxia/reoxygenation-induced injury in human cardiac tissue. miR-200 mediates the protective effects of erythropoietin on epithelial-mesenchymal transition in hypoxic HK-2 cells [9]. miR-429 has been shown to promote differentiation of osteoblastic cells by hypoxia [10]. Suppression of the hypoxia-induced apoptosis-stimulating protein p53-2 has been mainly attributed to elevated miR-205 levels in another study [11]. Strategies targeting miR-19b-3p may constitute a thought-provoking approach to ameliorate Japanese encephalitis virus-mediated inflammation [12]. According to recent studies, miR$19 \mathrm{~b}-3 \mathrm{p}$ is associated with various cancers, such as prostate cancer [13], gastric cancer [14], nasopharyngeal carcinoma [15], and lung adenocarcinoma [16]. Moreover, miR-19b-3p is a novel biomarker for the early diagnosis of acute myocardial infarction [17] and is related to primary myelofibrosis [18], Fragile X syndrome [19], hyperlipidemia and hepatic insulin resistance [20]. Based on these findings, miR-19b-3p plays a crucial role as a novel biomarker for the early diagnosis of different cancers induced by hypoxia.

We used a non-model bird species, the great tit (Parus major), which is widely distributed across the Eurasian continent encircling the central Asian desert and the QinghaiTibet Plateau (QTP), to determine whether any miRNAs contribute the genetic response to hypoxic conditions and identify their functional effects. In the southwestern mountains (SWM), the great tit is distributed between 2000 and 4500 meters above sea level. The highaltitude population of great tits shows an accelerated genetic selection for carbohydrate energy metabolism and the hypoxia response (e.g., the MAPK signaling pathway) compared to lowland populations [21]. Due to the crucial role of the MAPK signaling pathway in the hypoxia response in the great tit, we chose MAPK1 as a candidate gene. We hypothesized that miRNAs might play an essential role in regulating the MAPK1-mediated hypoxia response.

In this study, we first detected a set of differentially expressed miRNAs between highaltitude and low-altitude populations of great tit to examine this possibility. The result showed that MAPK1 bound only miR-19b-3p of the 14 miRNAs predicted by both TargetScan and miRanda software. We therefore validated the computational prediction of miR-19b-3p binding to the 3'UTR of MAPK1 using a luciferase reporter assay. We established the great tit embryonic fibroblast cell line and studied the relationship between miRNA-19b-3p and $M A P K 1$ in these cells.

\section{Materials and Methods}

\section{Sequencing of the miRNA transcriptome}

Tissue samples were obtained from the QTP (high altitude) and Beijing region (low altitude). The field collection of embryos was performed with the permission of the State Forestry Administration of China and conformed to the National Wildlife Conservation Law of China. All animals in this study were maintained in accordance with guidelines established by the Institute of Zoology for the care and use of experimental animals. Our experiments were also approved by the animal experimental and medical ethics committee of the Institute of Zoology, Chinese Academy of Sciences.

Total RNA was extracted using TRIzol (Sangon, Shanghai, China), according to the manufacturer's instructions. RNA concentrations and quality were estimated from the optical density measured at 260 and 


\section{Cellular Physiology Cell Physiol Biochem 2018;46:546-560

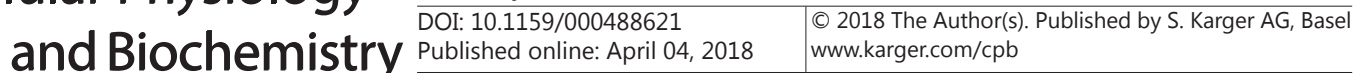

$280 \mathrm{~nm}$ using a UV spectrophotometer. Small RNA libraries were constructed using a TruSeq small RNA sample preparation kit (Illumina). Briefly, the 3' and 5' RNA adapters were ligated to the corresponding ends of small RNAs. Following adapter ligation, cDNAs were synthesized from all individual samples, according to the manufacturer's instructions (TaKaRa, RR014A, China). The cDNA products were PCR-amplified with index primers that were complementary to the ends of the adapter sequences. The PCR amplicons were sequenced on an Illumina HiSeq ${ }^{\mathrm{TM}} 2500 / \mathrm{MiSeq}$ sequencing system. The signature hairpin structure of miRNA precursor has been used to predict new miRNAs, and we integrated miREvo [22] and mirdeep2 [23] to analyze the new miRNAs. The miRNA read counts were normalized by transcripts per million (TPM) when comparing miRNA expression levels [24]. We identified differentially expressed genes from RNA-seq data using DESeq $[25,26]$.

\section{Computational prediction of miRNA targets}

Three established miRNA target prediction algorithms, TargetScan5.1, miRbase, and miRGene prediction analyses, were used to identify the candidate miRNAs that potentially targeted genes related to the hypoxia adaptation. Kyoto Encyclopedia of Genes and Genomes (KEGG) pathways were used to identify the metabolic pathways and signaling pathways in which the predicted target genes were involved [27]. We used the delta-delta Ct method to calculate fold changes [28].

\section{Validation using an in vitro luciferase assay}

Dual luciferase reporters were constructed. A fragment of the MAPK1 mRNA (XM_015644102.2) containing the predicted binding site for pma-miR-19b-3p was synthesized (Table 2) and subcloned downstream of the luciferase reporter gene in the psiCHECKTM-2 vector. MAPK1 mRNA 3'-UTR/mutant MAPK1 mRNA 3'-UTR and miR-19b-3p/negative control (nc) were co-transfected into 293T cells. The activities of the firefly and Renilla luciferases were measured $48 \mathrm{~h}$ after transfection with the Dual-Luciferase Reporter Assay System (Promega, E1910) using a luminometer (Promega). The results were expressed as the ratio of Renilla/firefly luciferase activity (mean \pm S.E.M.) from six independent replicates.

\section{Assessment of the co-localization of miR-19b-3p and its targets using in situ hybridization}

A combined two-color fluorescence in situ analysis of miRNA-19b-3p and MAPK1 localization in the heart tissue from the great tit was performed by co-labelling the miRNA and its target gene [29]. An antisense locked nucleic acid detection probe for miR-19b-3p was labeled with CY3, and the sense probe for MAPK1 was labeled with FITC. The heart tissue was fixed with $4 \%$ paraformaldehyde overnight. The paraffin-embedded integument tissue slides were deparaffinized in xylene, rehydrated with an ethanol gradient, digested with $20 \mu \mathrm{g} / \mathrm{ml}$ proteinase $\mathrm{K}$ at $37^{\circ} \mathrm{C}$ for $15 \mathrm{~min}$, and incubated with the locked nucleic acid miRNA probes and its target RNA probe at $60^{\circ} \mathrm{C}$ for $5 \mathrm{~min}$. The slides were then hybridized for 7-15 h at $37^{\circ} \mathrm{C}$ and washed with $0.2 \times \mathrm{SSC}$ and $2 \%$ BSA at $4^{\circ} \mathrm{C}$ for $5 \mathrm{~min}$. The signals of the miRNA-19b-3p and MAPK1 probes were detected using an LSM 710 confocal fluorescence microscope.

\section{Cell apoptosis and cell cycle detection}

The effects of miRNA-19b-3p and MAPK1 on cell apoptosis and cell cycle distribution were evaluated using the Annexin V-FITC/PI Apoptosis Assay Kit (Vazyme Biotech Co., Ltd, NJ, USA) and the Cell Cycle Detection Kit (KeyGen Biotech, Nanjing, China), respectively, according to the manufacturers' protocols. Cells were resuspended in $1 \times$ binding buffer to a final density of $1 \times 10^{5}$ to $5 \times 10^{5} \mathrm{cells} / \mathrm{ml}$. Five microliters of Annexin V-FITC were added to each $100 \mu \mathrm{l}$ of suspended cells, mixed and incubated for 20 min at room temperature in the dark. Then, $5 \mu$ l of the propidium iodide staining solution was added, mixed and incubated at RT in the dark for 5 min. Finally, $400 \mu \mathrm{l}$ of $1 \times$ binding buffer was added, mixed and analyzed on a flow cytometer (FACSCalibur, BD, Franklin Lakes, NJ, USA). The same method was used to analyze cell cycle profiles. Three replicated experiments were performed.

\section{Quantitative Real-Time PCR ( $q R T$-PCR)}

The prepared cDNAs were amplified with QuantiTect SYBR Green PCR Kit (Qiagen, 204143, Germany) and gene-specific primers in a final reaction volume of $25 \mu$ l. The primer sequences are listed in Table 1. Amplification and quantification were performed in an Exicycler 96 Quantitative PCR Analyzer (Bio-Rad Laboratories, USA) under a program of preincubation at $95^{\circ} \mathrm{C}$ for $10 \mathrm{~min}, 40$ cycles of denaturation at $95^{\circ} \mathrm{C}$ 
for $15 \mathrm{~s}$, annealing at $60^{\circ} \mathrm{C}$ for $30 \mathrm{~s}$, and extension at $72^{\circ} \mathrm{C}$ for 30 s. Melting curves for each gene were analyzed to ensure the amplification of single PCR products. $\beta$-Actin was used for normalization. Each experiment was independently repeated at least 3 times, and fold changes in gene expression were analyzed using the $2^{-\Delta \Delta \mathrm{Ct}}$ method.

\section{Western blotting analysis}

Cells were harvested, rinsed twice with PBS, lysed in a denaturing lysis buffer containing protease inhibitors (Total Protein Extraction Kit, KeyGen Biotech, Nanjing, China) for $30 \mathrm{~min}$ on ice, and centrifuged (12, $000 \mathrm{rpm})$ for $15 \mathrm{~min}$ at $4{ }^{\circ} \mathrm{C}$. Protein concentration of the lysates was determined using a BCA Protein Assay Kit (Vazyme Biotech, Nanjing, China). Proteins were separated on an 8\% sodium dodecyl sulfide-polyacrylamide gel and transferred to a polyvinylidene fluoride membrane (Merck Millipore). Membranes were blocked using 5\% non-fat dried milk in Tris-buffered saline with $0.1 \%$ Tween 20 (pH 7.6) for $1 \mathrm{~h}$ at room temperature and then incubated with a chip-grade MAPK1 (ERK2) antibody (1:200, R\&D, MAB1230-SP) overnight at $4{ }^{\circ} \mathrm{C}$. Membranes were then incubated with horseradish peroxidase-conjugated secondary antibodies for $1 \mathrm{~h}$ at room temperature. Horseradish peroxidase was detected using an enhanced chemiluminescence detection system (ECL Kit; Thermo Scientific, USA).

\section{MTT analysis}

Cell proliferation was measured with an MTT assay. Cells in each well of 96-well plates were treated with miR-19b-3p and MAPK1. After 0, 24, 48 and $72 \mathrm{~h}$ incubations, cells were treated with a $5 \mathrm{mg} / \mathrm{ml}$ MTT solution for $4 \mathrm{~h}$ at $37^{\circ} \mathrm{C}$. The MTT solution was discarded and formazan crystals were dissolved in $150 \mu \mathrm{l}$ of DMSO. Finally, the absorbance was measured at $490 \mathrm{~nm}$ using a microplate reader.

\section{Glucose assay}

To investigate whether MAPK1 regulates glucose metabolism during GEF growth under hypoxic conditions, glucose concentrations in media were measured using a colorimetric glucose assay kit (Sigma, US), according to the manufacturer' protocol.

\section{Statistical analysis}

All experimental data are presented as the mean \pm S.E.M. of three independent experiments, and $P<$ 0.05 was considered statistically significant. ANOVA and subsequent Tukey's post hoc tests were performed to determine the significance level of each effect. All statistical analyses were performed using SPSS 17.0.

\section{Results}

\section{Identification of miRNAs}

We sequenced transcriptomes of small RNAs in the heart tissue from both high- and lowaltitude great tits to identify the miRNAs associated with hypoxia adaptation in the species. Fourteen miRNAs related to the hypoxia response were differentially expressed between the high- and low-altitude great tits (Fig. 1A), with 8 up-regulated and 6 down-regulated miRNAs. Of these 14 miRNAs, two novel miRNAs (novel_4 and novel_57) were identified 
in the great tit compared to the chicken (Fig. 1B).

$\begin{aligned} & \text { Prediction of miRNA } \\ & \text { targets } \\ & \text { We examined the }\end{aligned}$
expression of target genes to identify the candidate miRNAs that potentially targeted genes related to the hypoxia adaptation. MAPK1 has been reported to be involved in accelerated selection in the highland great tit during the hypoxia adaptation [21]. MAPK1 only bound to miR$19 \mathrm{~b}-3 \mathrm{p}$ of the 14 miRNAs predicted by both TargetScan and miRanda software. The miRNA-target DEG networks were successfully integrated using Cytoscape software, and $M A P K 1$ was one of the targets genes in the miRNA-target network (Fig. 2).

Interaction and Colocalization of miR-19b-3p and MAPK1

We predicted putative seed match sites for miR-19b$3 p$ in the 3'UTR of MAPK1 to determine whether miR-19b$3 \mathrm{p}$ interacted with the target gene $M A P K 1$. Ahighly conserved binding site was identified (Fig. 3A). Compared to the mutant, the luciferase activity of the wild-type MAPK1-3'UTR decreased by approximately $27 \%$ in the great tit embryonic fibroblasts (GEFs), according to the results of the promoterdriven luciferase assay. The negative control construct with mutations in the 3'UTR of MAPK1 (MAPK1-3'UTR Mut) showed no obvious change in luciferase activity (Fig. 3B).

We used fluorescence in situ hybridization to analyze the expression of miR-19b-3p and its target and to determine whether miR-19b-3p co-localized with MAPK1 in the great tit heart. Notably, miR-19b-3p and MAPK1 were co-expressed in the great tit heart (Fig. 3C), suggesting a direct, spatially constrained interaction between miR-19b-3p and MAPK1.

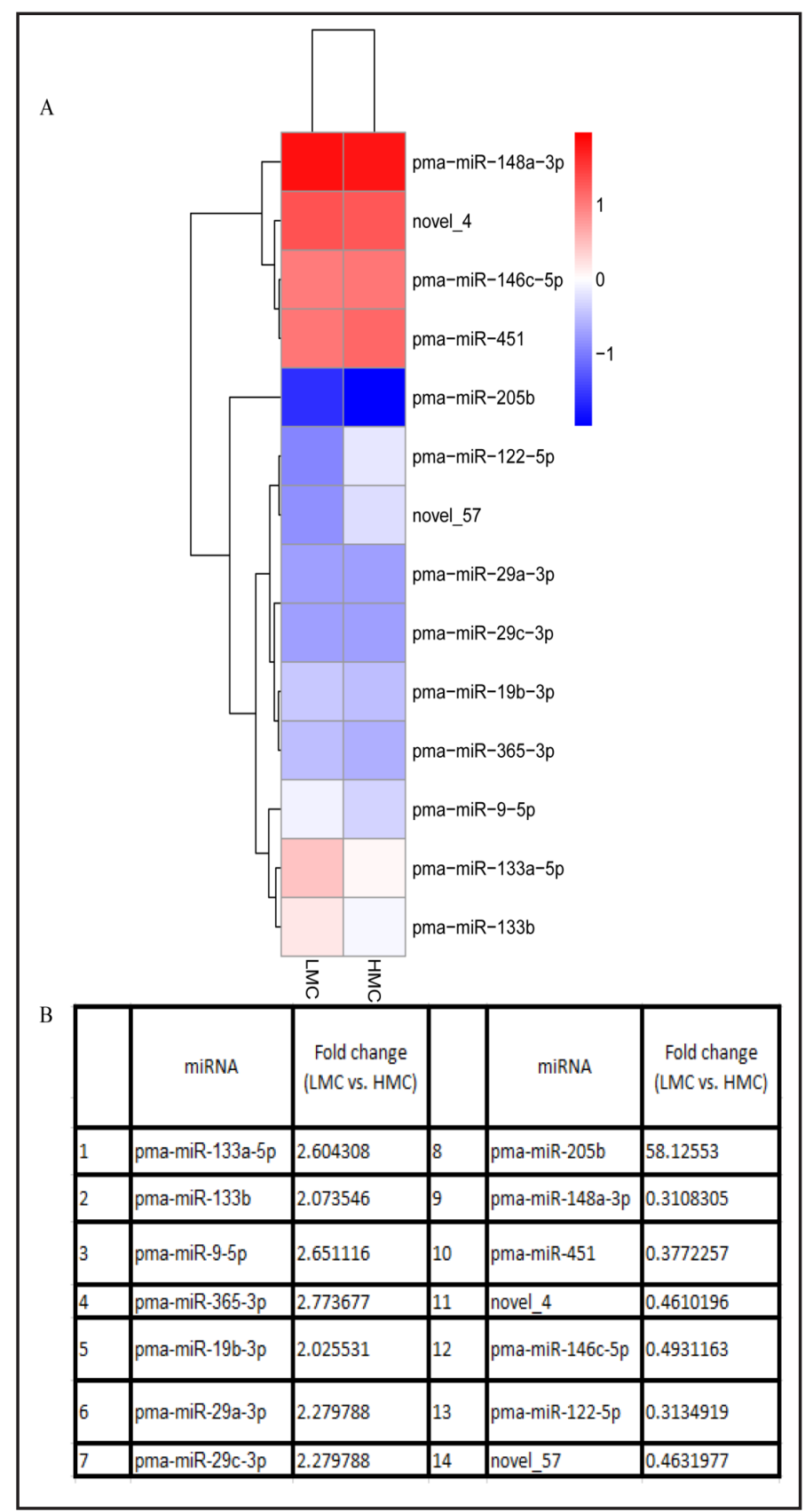

Fig. 1. miRNA identification. (A) Heatmap showing the clustering of miRNAs expressed in high altitude and low altitude great tits. Values for differentially expressed miRNAs in high-altitude male great tit cardiac tissues (HMC) and low-altitude male great tit cardiac tissues (LMC). The highly expressed miRNAs are highlighted in red. (B) Fold changes in the levels of differentially expressed miRNAs from HMC and LMC are presented in the table. 


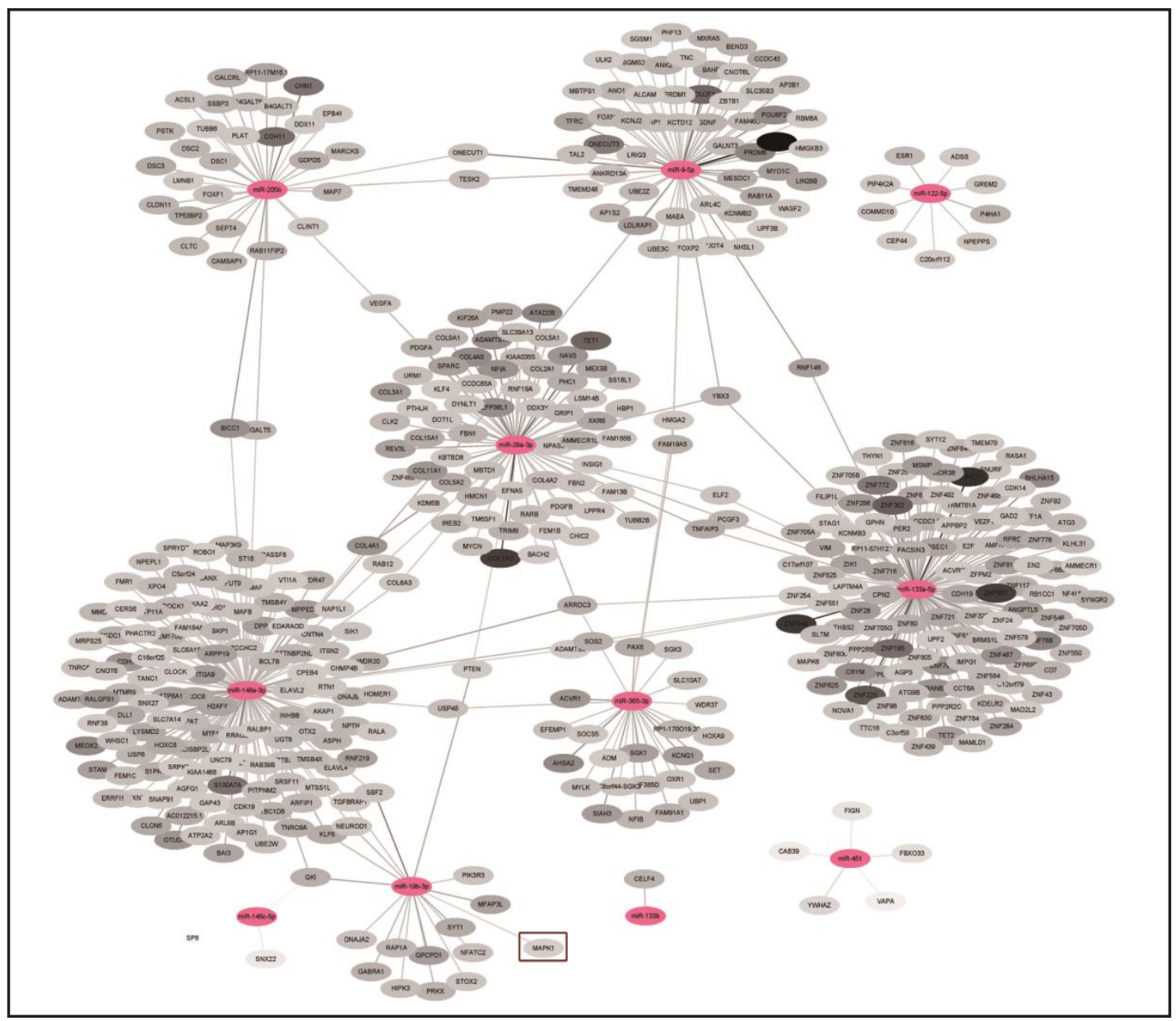

Fig. 2. Integrated miRNA-target regulatory network of DE miRNAs. Red circular nodes represent upregulated or down-regulated DE miRNAs, and gray circular nodes represent the predicted target genes for each miRNA. The size of the circular node denote the fold changes in miRNA expression. The target gene of miR-19b-3p, MAPK1, is labeled with a red box.

miR-19b-3p promotes GEF proliferation and endogenous mRNA expression under hypoxic conditions

GEFs were cultured in vitro to investigate the effect of miR-19b-3p on follicle growth. The effects of increased miR-19b-3p expression on cell proliferation were measured using the MTT assay. The miR-19b-3p mimics or inhibitors significantly inhibited proliferation under normoxic conditions, whereas the hypoxic conditions had a positive impact on cell proliferation (Fig. 4A). The miR-19b-3p mimics up-regulated the expression of miR-19b3p (Fig. 4B), whereas the inhibitors down-regulated miR-19b-3p expression 3.9-fold under normal conditions $(P<0.05$, Fig. $4 \mathrm{C})$. On the other hand, the mimics significantly up-regulated miR-19b-3p expression under hypoxic conditions ( $P<0.05$, Fig. 4B), and the inhibitors downregulated miR-19b-3p expression 1.4-fold (Fig. 4C).

miR-19b-3p regulates MAPK1 expression in GEFs

We treated GEFs with miR-19b-3p mimics or inhibitors to confirm that MAPK1 is the direct target gene of miR-19b-3p in GEFs. Overexpression of miR-19b-3p significantly down-regulated MAPK1 mRNA expression under normal conditions $(P<0.01)$; however, it significantly increased MAPK1 mRNA expression under hypoxic conditions (Fig. 5A). Furthermore, when miR-19b-3p activity in GEFs was transiently inhibited by a miR-19b- 
Fig. 3. Interaction and co-localization of miR-19b-3p and MAPK1. (A) Putative miR-19b-3p binding site in the 3'UTR of the MAPK1 mRNA. A mutation was generated in the putative miRNA binding site in the MAPK1-3'UTR sequence complementary to the seed region of miR-19b-3p, and cells were transfected with a wild-type or a mutant construct. (B) 3'UTR luciferase reporter assay in great tit embryonic fibroblasts after separate transfections with a pGL3promoter luciferase expression reporter vector containing the 3'UTR of each target gene, followed by treatment with miR-19b-3p mimics. The relative luciferase activity is reported as the ratio of Renilla/firefly luciferase activities. Data are presented as the mean \pm S.E.M. for multiple separate transfections $(n=3)$. ${ }^{*} \mathrm{P}<0.05$. (C) Co-localization of miR19b-3p (red fluorescence) and MAPK1 (green fluorescence) in low-altitude male great tit cardiac tissue.

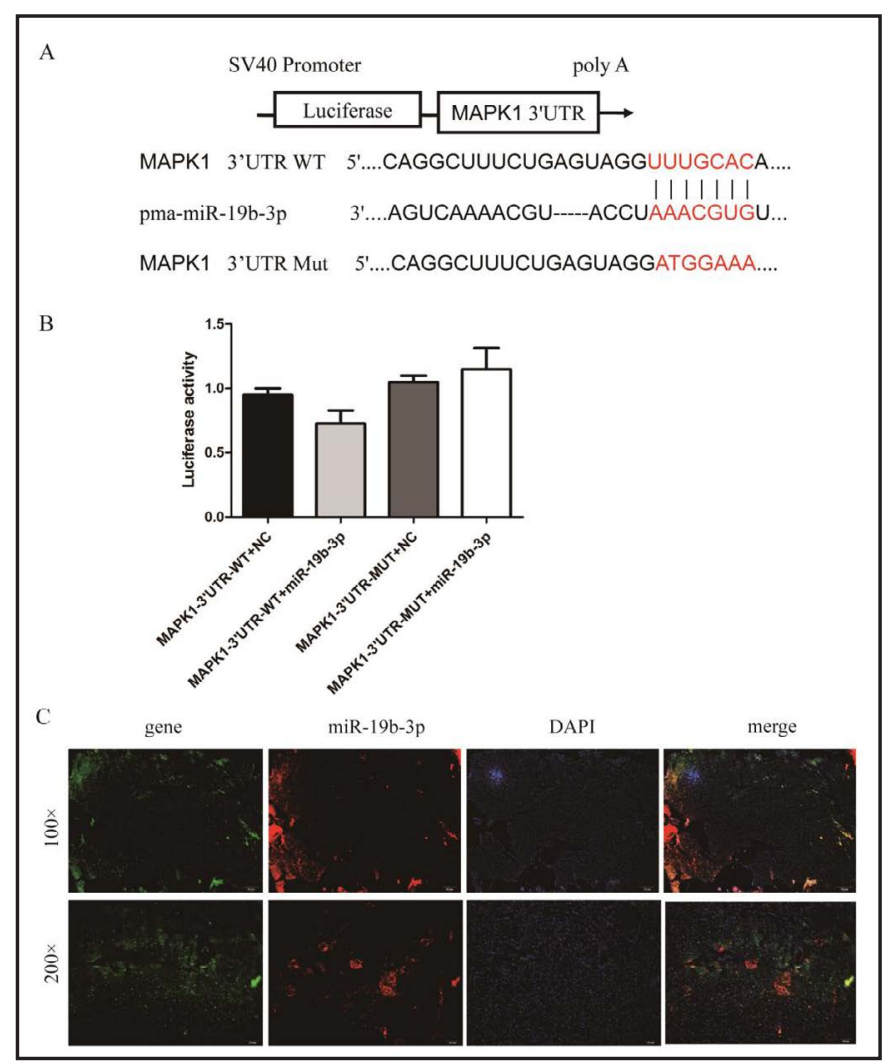

Fig. 4. miR-19b$3 p$ promotes GEF proliferation and endogenous mRNA expression under hypoxic conditions. (A) The effects of miR-19b-3p on GEF metabolic activity under normoxic and hypoxic conditions correlated by cell proliferation using the MTT assay. NC-mimics/inhibitor, negative control of the mimics/inhibitor, and the con-
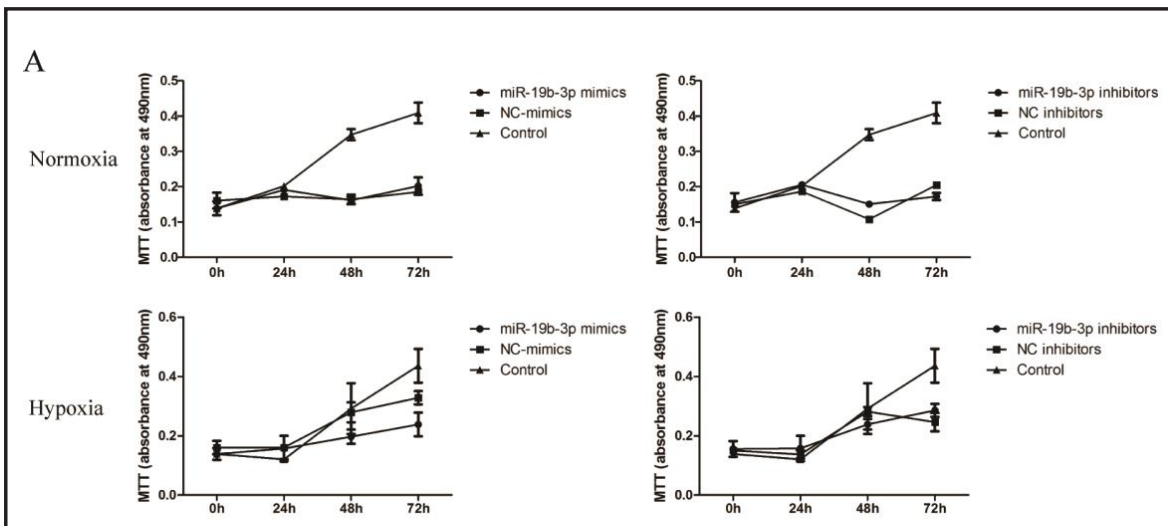

B
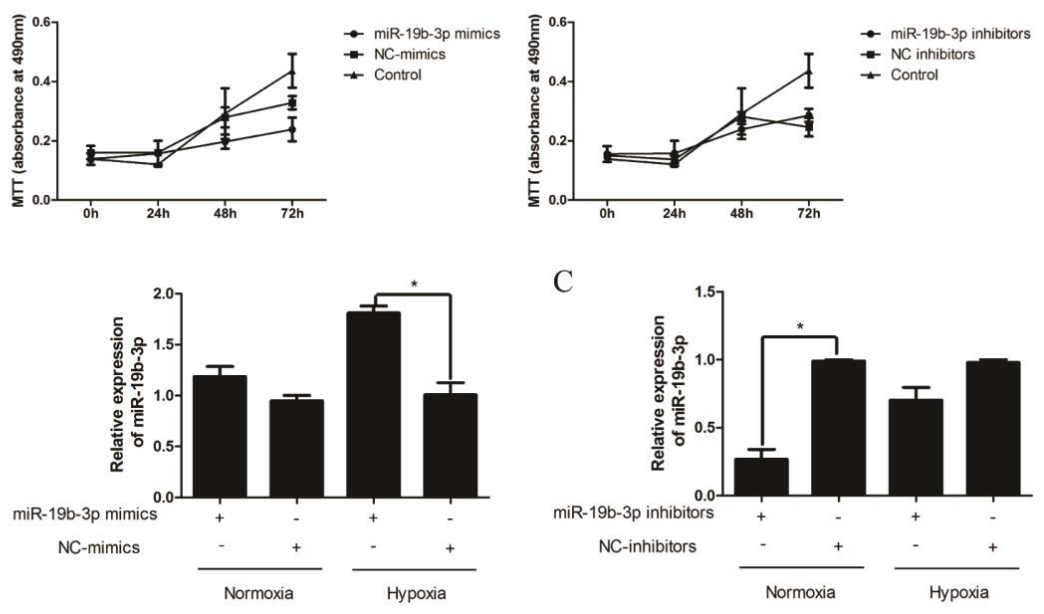

C

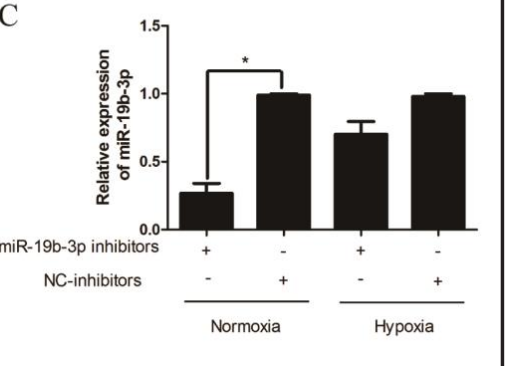
trol molecules

exhibit little homology to miRNAs. The efficiency of miR-19b-3p mimics (B) or inhibitors (C) in transfected GEFs grown under normoxic and hypoxic conditions for $24 \mathrm{~h}$. Data are presented as the mean \pm S.E.M. for multiple separate transfections $(\mathrm{n}=3)$. ${ }^{*} \mathrm{P}<0.05$. 
A

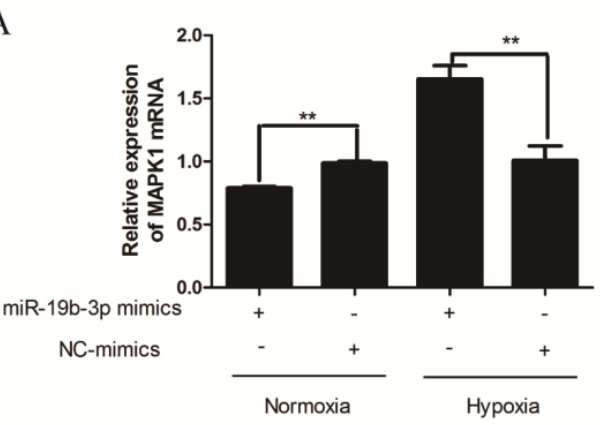

$\mathrm{C}$

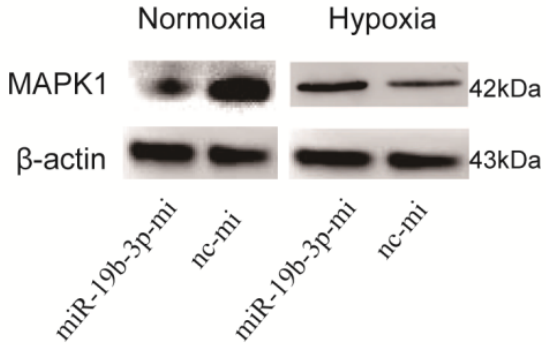

E

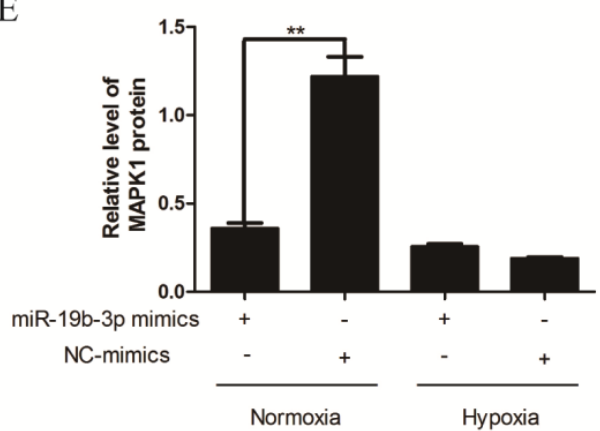

B

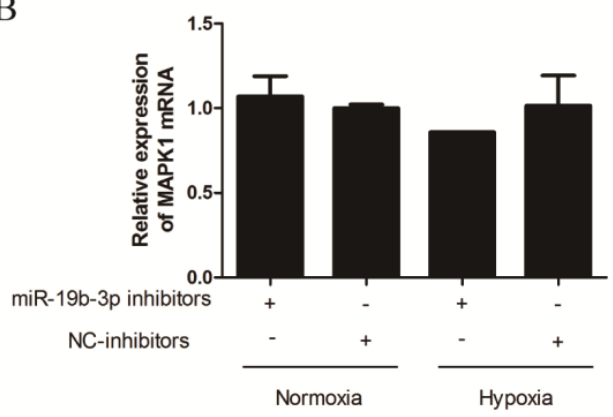

D

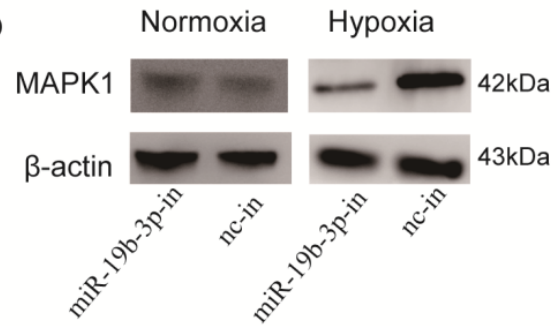

$\mathrm{F}$

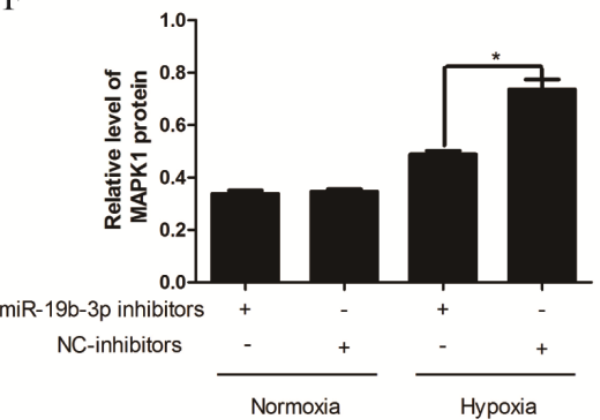

Fig. 5. miR-19b-3p regulates MAPK1 expression in GEFs. (A) Relative quantification of MAPK1 mRNA levels. GEFs were transfected with miR-19b-3p mimics and grown under normoxic and hypoxic conditions. NCmimics/inhibitor, negative control of the mimics/inhibitor, and the control molecules exhibit little homology to miRNAs. (B) Relative quantification of MAPK1 mRNA levels. GEFs were transfected with miR-19b-3p inhibitors and grown under normoxic and hypoxic conditions. (C) Analysis of MAPK1 protein levels. GEFs were transfected with miR-19b-3p mimics (miR-19b-3p-mi) and nc mimics (nc-mi), and protein extracts were analyzed by Western blotting. (D) Analysis of MAPK1 protein levels. GEFs were transfected with miR19b-3p inhibitors (miR-19b-3p-in) and nc inhibitors (nc-in), and protein extracts were analyzed by Western blotting. (E) Quantification of MAPK1 protein levels in cells overexpressing miR-19b-3p. (F) Quantification of MAPK1 protein levels in cells transfected with miR-19b-3p inhibitors. The results are presented as the mean \pm S.E.M. for multiple separate transfections $(\mathrm{n}=3) .{ }^{*} \mathrm{P}<0.01,{ }^{*} \mathrm{P}<0.05$.

$3 p$ inhibitor, the activation of MAPK1 expression by miR-19b-3p increased under normoxic conditions and reduced under hypoxic conditions (Fig. 5B). Levels of the MAPK1 protein were significantly decreased by the miR-19b-3p mimics under normoxic conditions $(P<0.01)$ but increased under hypoxic conditions (Fig. 5C, E). In contrast, the miR-19b-3p inhibitors had the reverse effect on the level of the MAPK1 under normoxic conditions and significantly decreased the level of the MAPK1 protein under hypoxic conditions $(P<0.05)$ (Fig. 5D and $5 \mathrm{~F})$. 


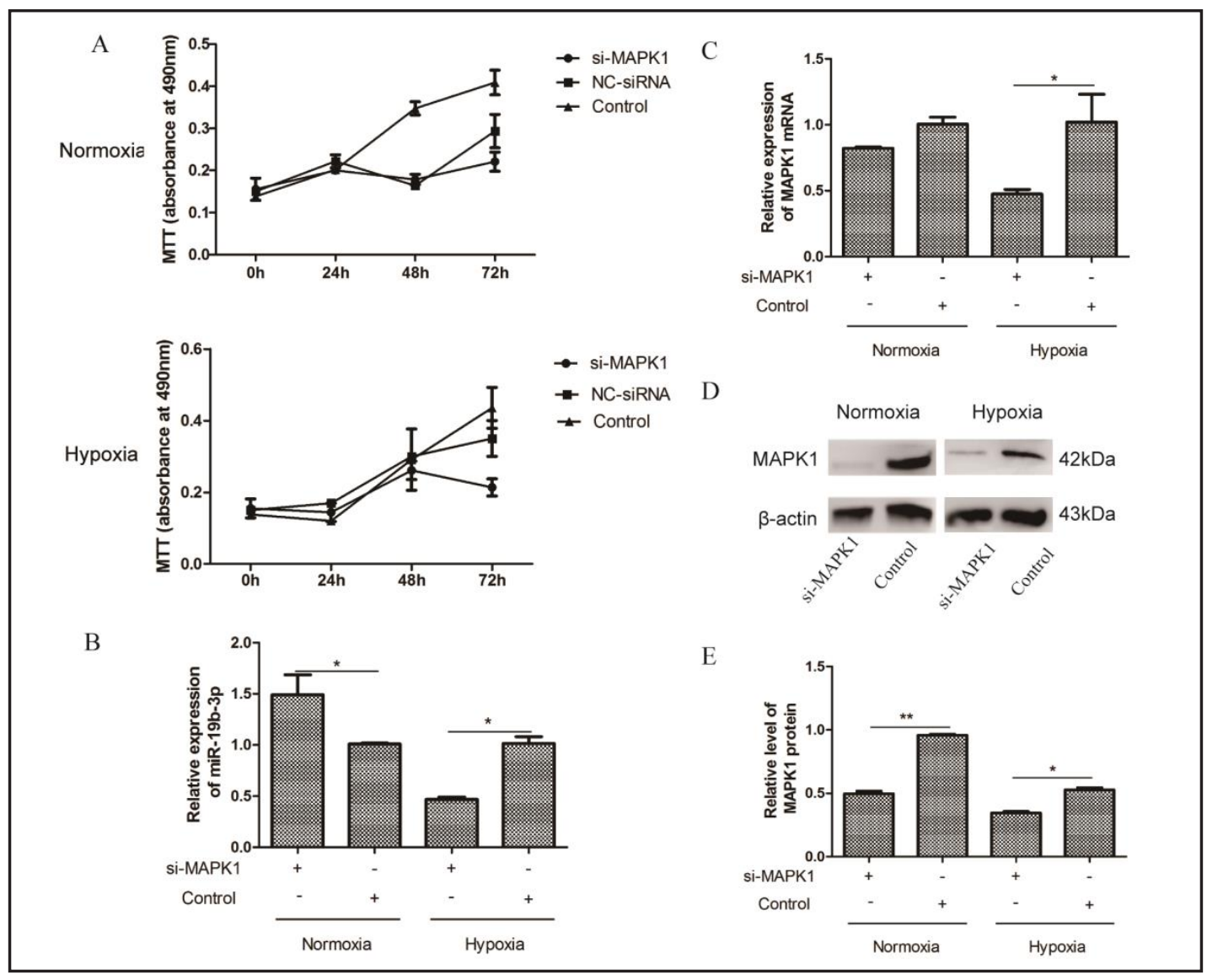

Fig. 6. MAPK1 is involved in GEF proliferation and quantification of MAPK1 mRNA and protein. (A) Effects of MAPK1 knockdown on GEF metabolic activity under normoxic and hypoxic conditions were determined using the MTT assay. NC-mimics/inhibitor, negative control of the mimics/inhibitor, and the control molecules exhibit little homology to miRNAs. (B) Quantification of miR-19b-3p mRNA levels $24 \mathrm{~h}$ after GEFs were transfected with the MAPK1 siRNA. (C) Quantification of MAPK1 mRNA levels $24 \mathrm{~h}$ after GEFs were transfected with the MAPK1 siRNA. The results are presented as the mean \pm S.E.M. for multiple separate transfections $(\mathrm{n}=3) .{ }^{*} \mathrm{P}<0.01,{ }^{*} \mathrm{P}<0.05$. (D) The expression levels of the MAPK1 protein were determined by Western blotting $48 \mathrm{~h}$ after GEFs were transfected with the MAPK1 siRNA. (E) Quantification of MAPK1 protein levels. The results are presented as the mean \pm S.E.M. for multiple separate transfections $(n=3)$. ${ }^{* *} \mathrm{P}<0.01,{ }^{*} \mathrm{P}<0.05$.

MAPK1 is involved in GEF proliferation and quantification of MAPK1 mRNA and protein

We transfected GEFs with a MAPK1 siRNA to investigate the effect of MAPK1 on follicle growth. The proliferation of cells transfected with si-MAPK1 was significantly increased under hypoxic conditions compared with normal conditions (Fig. 6A). The expression of miR$19 b-3 p$ was up-regulated when the cells were transfected with si-MAPK1 compared to the negative control siRNA under normoxic conditions $(P<0.05)$, and the opposite results were observed under hypoxic conditions (Fig. 6B). Under hypoxic conditions, MAPK1 knockdown significantly decreased the expression of the MAPK1 mRNA $(P<0.05)$ (Fig. 6C). The MAPK1 siRNA down-regulated MAPK1 protein expression under normal and $(P<0.01)$ (Fig. 6D) hypoxic conditions $(P<0.05)$ (Fig. 6E).

miR-19b-3p and MAPK1 induce GEF apoptosis and regulate the cell cycle under hypoxic conditions

We used the Annexin V-FITC/PI apoptosis assay and the Cell Cycle Detection Kit to investigate whether miR-19b-3p and MAPK1 regulate the cell cycle and apoptosis in GEFs. 
A
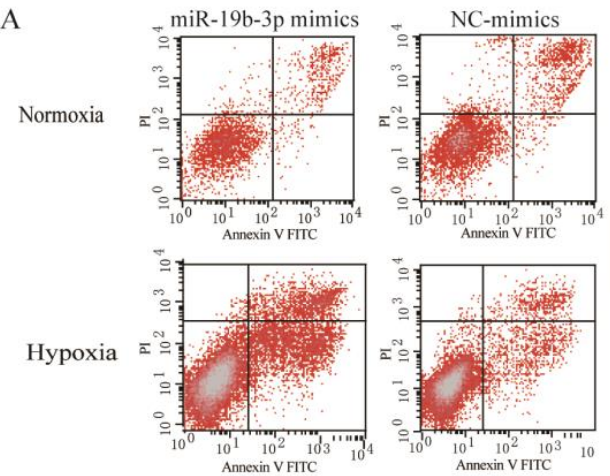

B

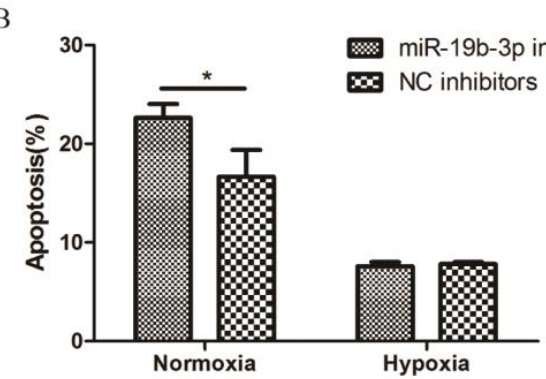

$\mathrm{D}$

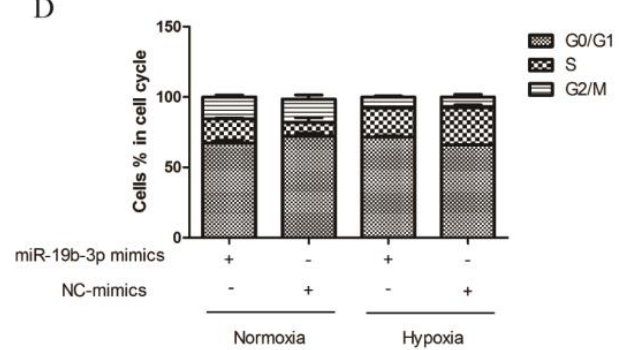

$\mathrm{F}$

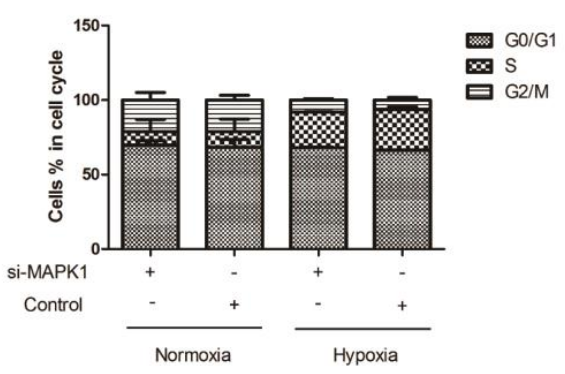

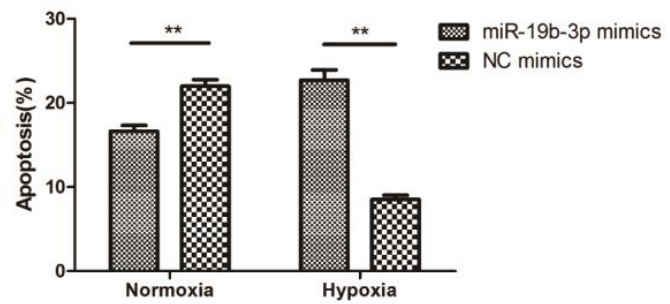

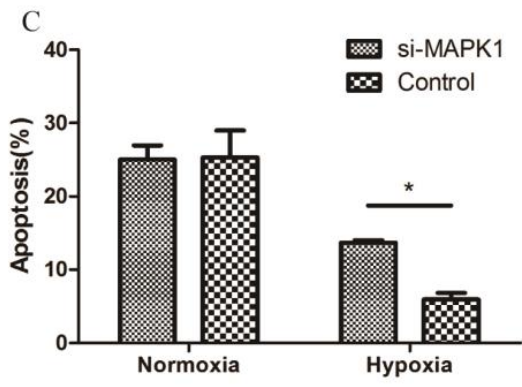

$\mathrm{E}$

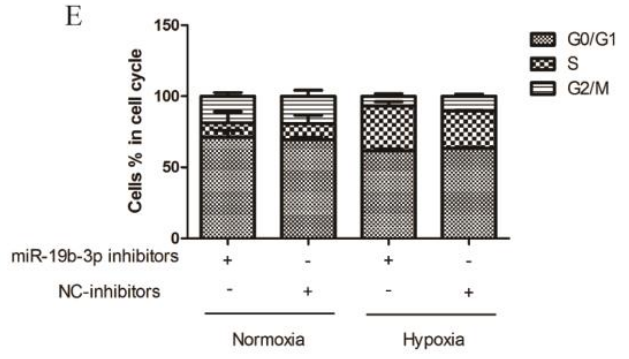

G

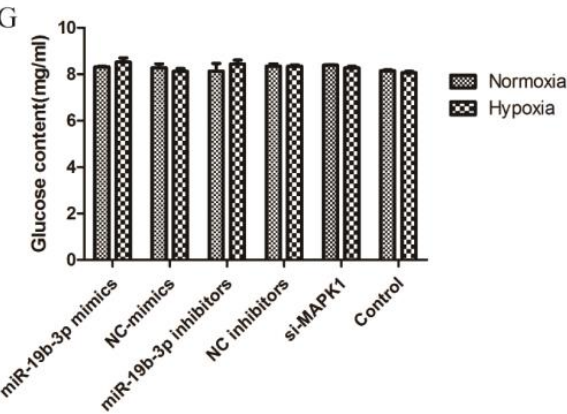

Fig. 7. miR-19b-3p and MAPK1 induce GEF apoptosis and regulate the cell cycle under hypoxic conditions. (A-C) Roles of miR-19b-3p and si-MAPK1 in GEF apoptosis and a representative set of flow cytometry images are shown. The apoptosis rate of GEFs was analyzed. Apoptotic cells are shown in the upper left, upper right, and lower right quadrants of each panel. Data are presented as the mean \pm S.E.M. for multiple separate transfections $(\mathrm{n}=3) .{ }^{*} \mathrm{P}<0.01,{ }^{*} \mathrm{P}<0.05$. NC-mimics/inhibitor, negative control of the mimics/inhibitor, and the control molecules exhibit little homology to miRNAs. (D-F) Cell cycle profiles were analyzed by flow cytometry. The cell cycle distribution was determined using FACS analysis. Data show the distribution of GEFs transfected with the three constructs in G1, S and G2 phases. The results are presented as the mean \pm S.E.M. for multiple separate transfections $(\mathrm{n}=3)$. ${ }^{* *} \mathrm{P}<0.01,{ }^{*} \mathrm{P}<0.05$. (G) Glucose levels were determined using a colorimetric glucose assay kit. 


\section{Cellular Physiology Cell Physiol Biochem 2018;46:546-560

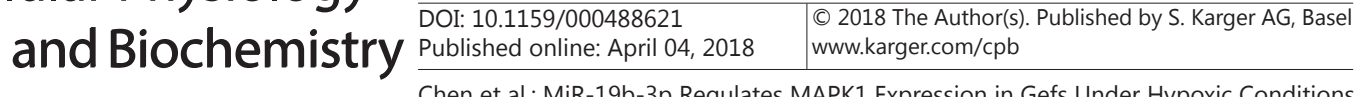

Cells treated with miR-19b-3p mimics exhibited a significant decrease in apoptosis under normoxic conditions $(P<0.01)$ and a significant increase under hypoxic conditions $(P<0.01$, Fig. 7A). No differences in the apoptosis rate were observed in GEFs transfected with the miR-19b-3p inhibitors (Fig. 7B). In contrast, the apoptosis rate was significantly increased in GEFs transfected with si-MAPK1 under hypoxic conditions $(P<0.05$, Fig. 7C). Analysis of the cell cycle revealed an increased percentage of cells in $S$ phrase in the miR-19b-3p mimics group compared with the nc mimics group under normoxic conditions (Fig. 7D). However, the percentages of cells in S phase decreased following treatment with miR-19b-3p mimics, inhibitors and si-MAPK1, and growth under normoxic conditions was comparable with that of cells grown under low-oxygen conditions (Fig. 7E and 7F).

\section{miR-19b-3p and MAPK1 do not affect glucose concentrations}

We used a colorimetric glucose assay to investigate whether miR-19b-3p and its target gene MAPK1 play roles in the responses to hypoxia that regulate glucose metabolism in GEFs. Glucose concentrations did not show significant changes in cells grown under either normal or hypoxic conditions (Fig. 7G).

\section{Discussion}

Involvement of miRNAs in the hypoxic response

The high-altitude population of the great tit has evolved a genetic adaptation that enables them to survive in a natural extremely hypoxic environment. According to a previous study, miRNAs related to hypoxia exhibit significant changes in Tibetan pigs compared to other species [30]. To date, very few miRNAs have been reported to play important roles in avian adaptation to hypoxia at high altitudes. Our results provide new evidence that miRNAs also affect biological processes related to the adaptation to hypoxia in great tits.

Our results show that many miRNAs were involved in the hypoxia adaptation, some of which have also been reported to be involved in hypoxia preconditioning in humans. For example, miR-29a-3p decreases hypoxia-induced pulmonary hypertension activity and inhibits pulmonary adventitial fibroblast activation [31]. Another miRNAs, miR-205, promotes the epithelial-mesenchymal transition process in response to hypoxia and may be a therapeutic biomarker of cervical and lung cancers [11]. We identified 14 differentially expressed miRNAs in cardiac muscle between high-altitude and low-altitude populations of great tits. These miRNAs participate in different signal transduction pathways and different functional gene networks, such as cell growth, immune response, and metabolism. Interestingly, according to a very recent study of human subjects, miR-365 was significantly up-regulated in trophoblasts under hypoxic conditions [32], consistent with the results of this study in great tits. As shown in the study by Calura et al., miR-19b-3p is also up-regulated in CD34+ cells in subjects with primary myelofibrosis and is associated with the hypoxia response [18]. Other reports have shown that miR-451 is up-regulated in human primary trophoblast cells in response to hypoxia [33] and in the lung during the development of pulmonary arterial hypertension after hypoxia [34]. Two of the up-regulated miRNAs identified in our study, miR-133a and miR-133b, were significantly increased in rats with chronic intermittent hypoxia-hypercapnia [35]. In contrast, a different result was observed for miR-133a, which is down-regulated in hypoxic H9c2 cells, and miR-133a also attenuates hypoxia-induced apoptosis [36].

In the current study, miR-19b-3p mimics significantly increased cell proliferation under hypoxic conditions compared to normoxic conditions. In previous studies, miR-19b has been shown to promote cardiac fibroblast proliferation and migration [37], improve cell survival and prevent $\mathrm{H}_{2} \mathrm{O}_{2}$-induced apoptosis in $\mathrm{H} 9 \mathrm{C} 2$ cardiomyocytes by targeting PTEN [38]. Thus, miR-19b overexpression might be a novel therapy for myocardial ischemia-reperfusion injury [38]. The expression of miR-19b was significantly induced in subjects with acute necrotizing pancreatitis, and miR-19b up-regulation promoted the necrosis of pancreatic 
acinar cells and vice versa [39]. TGF- $\beta 1 /$ Smad2 signaling is negatively regulated by miR$19 a-3 p / 19 b-3 p$, and these miRNAs inhibit the autophagy-mediated fibrosis of human cardiac fibroblasts by targeting TGF- $\beta$ RII [40]. In addition, miR-19b reduces hepatic stellate cell proliferation by targeting GRB2 in hepatic fibrosis models both in vitro and in vivo [41] and markedly diminishes liver fibrosis in rats and humans [42]. Hypoxia is also a basic pathological challenge associated with numerous cardiovascular disorders. For example, miR-101 expression protects cardiac fibroblasts from hypoxia-induced apoptosis [43]. In a rat hypoxia model, miR-122 and its target genes play roles in the hypoxia responses that regulate glucose and energy metabolism and serve as potential biomarkers of mechanical asphyxia [44]. All these results support the concept that miRNAs regulate the response to hypoxia.

\section{miR-19b-3p and its target gene MAPK1}

Our results indicate that one of the identified hypoxia-related miRNAs, miR-19b-3p, and its target MAPK1, are related to hypoxia adaptation. This conclusion is supported by both the microarray findings in samples from normal and hypoxic conditions, and the cell-based validation assays. The expression of the MAPK1 gene was regulated by miR-19b-3p under hypoxic conditions. MAPK signaling is an important pathway by which hypoxia promotes tumorigenesis by suppressing the transactivation activity of p300 [45], and thus plays an important role in tumor growth and transformation, depending on angiogenesis and alterations in glucose metabolism [46]. As shown in the study by Zhang et al [30]., the MAPK signaling pathway regulates adaptations to hypoxia in the Tibetan pig. In subsequent studies, MAPK1 (ERK) inhibitors completely prevented the inhibitory effect of high glucose on human umbilical vein endothelial cells [47]. However, significant changes in glucose concentrations were not observed in cultures grown under either normal or hypoxic conditions in our study. Further studies are required to illuminate the mechanism by which MAPK1 regulates glucose metabolism during great tit growth under hypoxic conditions. Based on the results from reporter assays and qPCR, MAPK1 is a direct target of miR-19b-3p, and miR-19b-3p overexpression increased the expression of the MAPK1 protein. The miR-19b-3p mimics significantly prevented the cell proliferation under normoxic conditions.

Our study for the first time identified 14 hypoxia response miRNAs that were significantly differentially expressed in cardiac tissues between great tits from the high and low altitudes. Considering that the MAPK signaling pathway plays a crucial role in the hypoxia response in the great tit, we chose MAPK1 as the candidate gene. Of the 14 miRNAs predicted by both TargetScan and miRanda software MAPK1 binds only to miR-19b-3p. We verified their functions and their interaction. Our results highlight the important mechanisms by which miRNAs regulate the avian high-altitude adaptation in a non-model bird species. Our findings lay the foundation for studies of miRNA-mediated mechanisms underlying hypoxic adaptation in other fauna.

\section{Acknowledgements}

We thank Enkui Duan for providing the cell culture conditions for the study. The project was funded by the Strategic Priority Research Programme of the Chinese Academy of Sciences (XDB13020300), the State Key Programme of NSFC $(31330073,31630069)$ to F.L., and the Key Laboratory of the Zoological Systematics and Evolution of the Chinese Academy of Sciences (No. Y229YX5105).

\section{Disclosure Statement}

The authors declare that they have no conflicts of interest. 


\section{Cellular Physiology Cell Physiol Biochem 2018;46:546-560 \begin{tabular}{l|l} 
and Biochemistry & DOI: 10.1159/000488621 \\
Publisno 2018 The Author(s). Published by S. Karger AG, Basel \\
www.karger.com/cpb
\end{tabular}

\section{References}

1 Bartel DP: Micrornas: Genomics, biogenesis, mechanism, and function. Cell 2004;116:281-297.

2 Forman JJ, Coller HA: The code within the code: Micrornas target coding regions. Cell Cycle 2010;9:15331541.

- 3 Li SS, Ran YJ, Zhang DD, Li SZ, Zhu D: Microrna-190 regulates hypoxic pulmonary vasoconstriction by targeting a voltage-gated k(+) channel in arterial smooth muscle cells. J Cell Biochem 2014;115:1196-1205.

-4 Zhi F, Shao N, Xue L, Xu Y, Kang X, Yang Y, Xia Y: Characteristic microrna expression induced by delta-opioid receptor activation in the rat liver under prolonged hypoxia. Cell Physiol Biochem 2017;44:2296-2309.

5 Zhi F, Xue L, Shao N, Deng D, Kang X, Chao D, Xu Y, Wang R, Yang Y, Xia Y: Delta-opioid receptor activation and microrna expression in the rat heart under prolonged hypoxia. Cell Physiol Biochem 2016;39:11181128.

6 Yang HH, Chen Y, Gao CY, Cui ZT, Yao JM: Protective effects of microrna-126 on human cardiac microvascular endothelial cells against hypoxia/reoxygenation-induced injury and inflammatory response by activating pi3k/akt/enos signaling pathway. Cell Physiol Biochem 2017;42:506-518.

7 Gong LC, Xu HM, Guo GL, Zhang T, Shi JW, Chang C: Long non-coding rna h19 protects h9c2 cells against hypoxia-induced injury by targeting microrna-139. Cell Physiol Biochem 2017;44:857-869.

8 Yan X, Liu J, Wu H, Liu Y, Zheng S, Zhang C, Yang C: Impact of mir-208 and its target gene nemo-like kinase on the protective effect of ginsenoside rb1 in hypoxia/ischemia injuried cardiomyocytes. Cell Physiol Biochem 2016;39:1187-1195.

-9 Bai J, Xiao X, Zhang X, Cui H, Hao J, Han J, Cao N: Erythropoietin inhibits hypoxia-induced epithelial-tomesenchymal transition via upregulation of mir-200b in hk-2 cells. Cell Physiol Biochem 2017;42:269-280.

10 Huang J, Peng J, Cao G, Lu S, Liu L, Li Z, Zhou M, Feng M, Shen H: Hypoxia-induced microrna-429 promotes differentiation of mc3t3-e1 osteoblastic cells by mediating zfpm2 expression. Cell Physiol Biochem 2016;39:1177-1186.

-11 Wang X, Yu M, Zhao K, He M, Ge W, Sun Y, Wang Y, Sun H, Hu Y: Upregulation of mir-205 under hypoxia promotes epithelial-mesenchymal transition by targeting aspp2. Cell Death Dis 2016;7:e2517.

12 Ashraf U, Zhu B, Ye J, Wan S, Nie Y, Chen Z, Cui M, Wang C, Duan X, Zhang H, Chen H, Cao S: Microrna-19b-3p modulates japanese encephalitis virus-mediated inflammation via targeting rnf11. J Virol 2016;90:47804795 .

13 Osip'yants AI, Knyazev EN, Galatenko AV, Nyushko KM, Galatenko VV, Shkurnikov MY, Alekseev BY: Changes in the level of circulating hsa-mir-297 and hsa-mir-19b-3p mirna are associated with generalization of prostate cancer. Bull Exp Biol Med 2017;162:379-382.

14 Zhang J, Song Y, Zhang C, Zhi X, Fu H, Ma Y, Chen Y, Pan F, Wang K, Ni J, Jin W, He X, Su H, Cui D: Circulating mir-16-5p and mir-19b-3p as two novel potential biomarkers to indicate progression of gastric cancer. Theranostics 2015;5:733-745.

15 Huang T, Yin L, Wu J, Gu JJ, Wu JZ, Chen D, Yu HL, Ding K, Zhang N, Du MY, Qian LX, Lu ZW, He X: Microrna19b-3p regulates nasopharyngeal carcinoma radiosensitivity by targeting tnfaip3/nf-kappab axis. J Exp Clin Cancer Res 2016;35:188.

16 Zhou X, Wen W, Shan X, Zhu W, Xu J, Guo R, Cheng W, Wang F, Qi LW, Chen Y, Huang Z, Wang T, Zhu D, Liu P, Shu Y: A six-microrna panel in plasma was identified as a potential biomarker for lung adenocarcinoma diagnosis. Oncotarget 2017;8:6513-6525.

17 Wang KJ, Zhao X, Liu YZ, Zeng QT, Mao XB, Li SN, Zhang M, Jiang C, Zhou Y, Qian C, Feng KG, Guan HQ Tang TT, Cheng X, Chen ZJ: Circulating mir-19b-3p, mir-134-5p and mir-186-5p are promising novel biomarkers for early diagnosis of acute myocardial infarction. Cell Physiol Biochem 2016;38:1015-1029.

18 Calura E, Pizzini S, Bisognin A, Coppe A, Sales G, Gaffo E, Fanelli T, Mannarelli C, Zini R, Norfo R, Pennucci V, Manfredini R, Romualdi C, Guglielmelli P, Vannucchi AM, Bortoluzzi S: A data-driven network model of primary myelofibrosis: Transcriptional and post-transcriptional alterations in cd34+ cells. Blood Cancer J 2016;6:e439.

19 Ma Y, Tian S, He S, Chen Q, Wang Z, Xiao X, Fu L, Lei X: The mechanism of action of fxr1p-related mir-19b-3p in sh-sy5y. Gene 2016;588:62-68.

20 Sud N, Zhang H, Pan K, Cheng X, Cui J, Su Q: Aberrant expression of microrna induced by high-fructose diet: Implications in the pathogenesis of hyperlipidemia and hepatic insulin resistance. J Nutr Biochem 2017;43:125-131. 


\section{Cellular Physiology Cell Physiol Biochem 2018;46:546-560 and Biochemistry \begin{tabular}{l|l} 
DOI: 10.1159/000488621 & $\begin{array}{l}\text { C } 2018 \text { The Author(s). Published by S. Karger AG, Basel } \\
\text { www.karger.com/cpb }\end{array}$
\end{tabular}

21 Qu Y, Tian S, Han N, Zhao H, Gao B, Fu J, Cheng Y, Song G, Ericson PG, Zhang YE, Wang D, Quan Q Jiang Z, Li $\mathrm{R}$, Lei F: Genetic responses to seasonal variation in altitudinal stress: Whole-genome resequencing of great tit in eastern himalayas. Sci Rep 2015;5:14256.

22 Wen M, Shen Y, Shi S, Tang T: Mirevo: An integrative microrna evolutionary analysis platform for nextgeneration sequencing experiments. BMC Bioinformatics 2012;13:140.

-23 Friedlander MR, Mackowiak SD, Li N, Chen W, Rajewsky N: Mirdeep2 accurately identifies known and hundreds of novel microrna genes in seven animal clades. Nucleic Acids Res 2012;40:37-52.

-24 Zhou L, Chen J, Li Z, Li X, Hu X, Huang Y, Zhao X, Liang C, Wang Y, Sun L, Shi M, Xu X, Shen F, Chen M, Han Z, Peng Z, Zhai Q, Chen J, Zhang Z, Yang R, Ye J, Guan Z, Yang H, Gui Y, Wang J, Cai Z, Zhang X: Integrated profiling of micrornas and mrnas: Micrornas located on xq27.3 associate with clear cell renal cell carcinoma. PLoS One 2010;5:e15224.

25 Love MI, Huber W, Anders S: Moderated estimation of fold change and dispersion for rna-seq data with deseq2. Genome Biol 2014;15:550.

26 Wang L, Feng Z, Wang X, Wang X, Zhang X: Degseq: An r package for identifying differentially expressed genes from rna-seq data. Bioinformatics 2010;26:136-138.

27 Kanehisa M, Araki M, Goto S, Hattori M, Hirakawa M, Itoh M, Katayama T, Kawashima S, Okuda S, Tokimatsu T, Yamanishi Y: Kegg for linking genomes to life and the environment. Nucleic Acids Res 2008;36:D480-484.

28 Livak KJ, Schmittgen TD: Analysis of relative gene expression data using real-time quantitative pcr and the 2(-delta delta c(t)) method. Methods 2001;25:402-408.

29 Nuovo GJ, Elton TS, Nana-Sinkam P, Volinia S, Croce CM, Schmittgen TD: A methodology for the combined in situ analyses of the precursor and mature forms of micrornas and correlation with their putative targets. Nat Protoc 2009;4:107-115.

-30 Zhang B, Qiangba Y, Shang P, Wang Z, Ma J, Wang L, Zhang H: A comprehensive microrna expression profile related to hypoxia adaptation in the tibetan pig. PLoS One 2015;10:e0143260.

-31 Luo Y, Dong HY, Zhang B, Feng Z, Liu Y, Gao YQ, Dong MQ, Li ZC: Mir-29a-3p attenuates hypoxic pulmonary hypertension by inhibiting pulmonary adventitial fibroblast activation. Hypertension 2015;65:414-420.

-32 Mori A, Nishi H, Sasaki T, Nagamitsu Y, Kawaguchi R, Okamoto A, Kuroda M, Isaka K: Hla-g expression is regulated by mir-365 in trophoblasts under hypoxic conditions. Placenta 2016;45:37-41.

33 Mouillet JF, Chu T, Hubel CA, Nelson DM, Parks WT, Sadovsky Y: The levels of hypoxia-regulated micrornas in plasma of pregnant women with fetal growth restriction. Placenta 2010;31:781-784.

-34 Caruso P, MacLean MR, Khanin R, McClure J, Soon E, Southgate M, MacDonald RA, Greig JA, Robertson KE, Masson R, Denby L, Dempsie Y, Long L, Morrell NW, Baker AH: Dynamic changes in lung microrna profiles during the development of pulmonary hypertension due to chronic hypoxia and monocrotaline. Arterioscler Thromb Vasc Biol 2010;30:716-723.

-35 Pan LL, Ke JQ Zhao CC, Huang SY, Shen J, Jiang XX, Wang XT: Electrical stimulation improves rat muscle dysfunction caused by chronic intermittent hypoxia-hypercapnia via regulation of mirna-related signaling pathways. PLoS One 2016;11:e0152525.

-36 Li AY, Yang Q, Yang K: Mir-133a mediates the hypoxia-induced apoptosis by inhibiting tagln2 expression in cardiac myocytes. Mol Cell Biochem 2015;400:173-181.

-37 Zhong C, Wang K, Liu Y, Lv D, Zheng B, Zhou Q, Sun Q, Chen P, Ding S, Xu Y, Huang H: Mir-19b controls cardiac fibroblast proliferation and migration. J Cell Mol Med 2016;20:1191-1197.

-38 Xu J, Tang Y, Bei Y, Ding S, Che L, Yao J, Wang H, Lv D, Xiao J: Mir-19b attenuates h2o2-induced apoptosis in rat h9c2 cardiomyocytes via targeting pten. Oncotarget 2016;7:10870-10878.

-39 Hu MX, Zhang HW, Fu Q, Qin T, Liu CJ, Wang YZ, Tang Q Chen YX: Functional role of microrna-19b in acinar cell necrosis in acute necrotizing pancreatitis. J Huazhong Univ Sci Technolog Med Sci 2016;36:221-225.

40 Zou M, Wang F, Gao R, Wu J, Ou Y, Chen X, Wang T, Zhou X, Zhu W, Li P, Qi LW, Jiang T, Wang W, Li C, Chen J, He $Q$, Chen Y: Autophagy inhibition of hsa-mir-19a-3p/19b-3p by targeting tgf-beta $r$ ii during tgf-beta1induced fibrogenesis in human cardiac fibroblasts. Sci Rep 2016;6:24747.

41 Ge S, Xie J, Liu F, He J, He J: Microrna-19b reduces hepatic stellate cell proliferation by targeting grb2 in hepatic fibrosis models in vivo and in vitro as part of the inhibitory effect of estradiol. J Cell Biochem 2015;116:2455-2464.

42 Lakner AM, Steuerwald NM, Walling TL, Ghosh S, Li T, McKillop IH, Russo MW, Bonkovsky HL, Schrum LW: Inhibitory effects of microrna $19 \mathrm{~b}$ in hepatic stellate cell-mediated fibrogenesis. Hepatology 2012;56:300310. 


\section{Cellular Physiology Cell Physiol Biochem 2018;46:546-560 \begin{tabular}{l|l} 
DOI: 10.1159/000488621 & $\begin{array}{l}\text { O 2018 The Author(s). Published by S. Karger AG, Basel } \\
\text { www.karger.com/cpb }\end{array}$ \\
\hline
\end{tabular}}

Chen et al.: MiR-19b-3p Regulates MAPK1 Expression in Gefs Under Hypoxic Conditions

43 Zhao X, Wang K, Liao Y, Zeng Q, Li Y, Hu F, Liu Y, Meng K, Qian C, Zhang Q, Guan H, Feng K, Zhou Y, Du Y, Chen Z: Microrna-101a inhibits cardiac fibrosis induced by hypoxia via targeting tgfbetari on cardiac fibroblasts. Cell Physiol Biochem 2015;35:213-226.

44 Zeng Y, Lv Y, Tao L, Ma J, Zhang H, Xu H, Xiao B, Shi Q, Ma K, Chen L: G6pc3, aldoa and cs induction accompanies mir-122 down-regulation in the mechanical asphyxia and can serve as hypoxia biomarkers. Oncotarget 2016;7:74526-74536.

45 Sang N, Stiehl DP, Bohensky J, Leshchinsky I, Srinivas V, Caro J: Mapk signaling up-regulates the activity of hypoxia-inducible factors by its effects on p300. J Biol Chem 2003;278:14013-14019.

-46 Folkman J: Angiogenesis in cancer, vascular, rheumatoid and other disease. Nat Med 1995;1:27-31.

-47 Kim MH, Kang HM, Kim CE, Han S, Kim SW: Ramipril inhibits high glucose-stimulated up-regulation of adhesion molecules via the erk1/2 mapk signaling pathway in human umbilical vein endothelial cells. Cell Mol Biol Lett 2015;20:937-947. 